\title{
The Vascular Flora of Tetraclinis Ecosystem in the Moroccan Central Plateau
}

\author{
Youssef Dallahi \\ Driss Chahhou \\ Laboratory for Physical Geography, Department of Geography, \\ Faculty of Arts and Humanities, \\ Mohammed V University, Rabat, Morocco \\ Abderrahman Aafi \\ National Forestry Engineering School Salé, Morocco \\ Mohamed Fennane \\ Scientific Institute, Mohammed V University, Rabat, Morocco
}

Doi: 10.19044/esj.2017.v13n33p104 URL:http://dx.doi.org/10.19044/esj.2017.v13n33p104

\begin{abstract}
The main objective of this study is to quantify the floral richness and diversity of Tetraclinis ecosystem in the Moroccan Central Plateau. The approach was based on over 300 floristic surveys covering the different parts of the Moroccan Central Plateau forests. It also entails the analysis and processing of data from studies in the region. The results indicate that there are 233 taxa belonging to 56 families.
\end{abstract}

Keywords: Floral richness, Tetraclinis ecosystem, Moroccan Central Plateau

\section{Introduction}

Due to its typical and geographical position between the Atlantic Ocean to the west and the Mediterranean Sea to the north, Morocco is characterized by high vascular plant diversity with approximately 4200 species and subspecies belonging to 135 families and 940 genera (Benabid, 2000). The endemic flora includes 951 species and subspecies, representing $21 \%$ of the Moroccan vascular plants. The richest floristic regions for endemic species are located at the top of high mountains.

By its geographical position, its varied topography, geology, ecoregion and climate, the Central Plateau of Morocco includes a large area of forest ecosystems with an important floristic diversity. However, this flora richness is still not well known and botanical studies within the zone still remains limited. 
Therefore, this study aims to quantify and characterize the floristic richness and diversity of Tetraclinis ecosystem in the Moroccan Central Plateau. This study was carried out for the purpose of better knowledge and to highlight the floristic potentialities of the region to support sustainable management biodiversity.

\section{Study Area}

Moroccan Meseta or Moroccan Central Plateau (Figure 1) is an old massive area located in the north-west of Morocco. It covers a wide range of highlands at altitudes between 500 and 1250 meters, extending from the Atlantic Ocean to the west until the Middle Atlas to the east and the Phosphate plateau to the south.

The area is geologically characterized by an immense diversity with the dominance of primary and quaternary structures. However, the schists, sandstone, and basalt from the quaternary are well represented (Combe $e t$ al., 1975). In terms of pedology, the Central Plateau region is marked by the soils diversity (rendzinas, brown forest soils, lithosols and regosols). However, due to the steep slopes, lithosols are by far, the most frequent type of soils (Beaudet, 1969).

The climate is Mediterranean with an average annual rainfall ranging from 350 to $1000 \mathrm{~mm}$. Average low temperatures vary from $0.9^{\circ} \mathrm{C}$ to $6{ }^{\circ} \mathrm{C}$ and average high temperatures vary from $29{ }^{\circ} \mathrm{C}$ to $35^{\circ} \mathrm{C}$ (Combe et al., 1975). Bioclimatic zoning shows the existence of four types of climate: humid, sub-humid, semi-arid, and arid (Combe et al., 1975).

The vegetation study of the area revealed a diversity in terms of species involved in the individualization of forest ecosystems. The major species encountered are Tetraclinis articulata, Quercus rotundifolia, and Quercus suber.

Furthermore, the Moroccan Central Plateau showcases an outstanding biological diversity through the presence of a large number of plant and animal species, and the existence of several Sites of Biological and Ecological Interest (SBEI) that reflect this magnitude. 


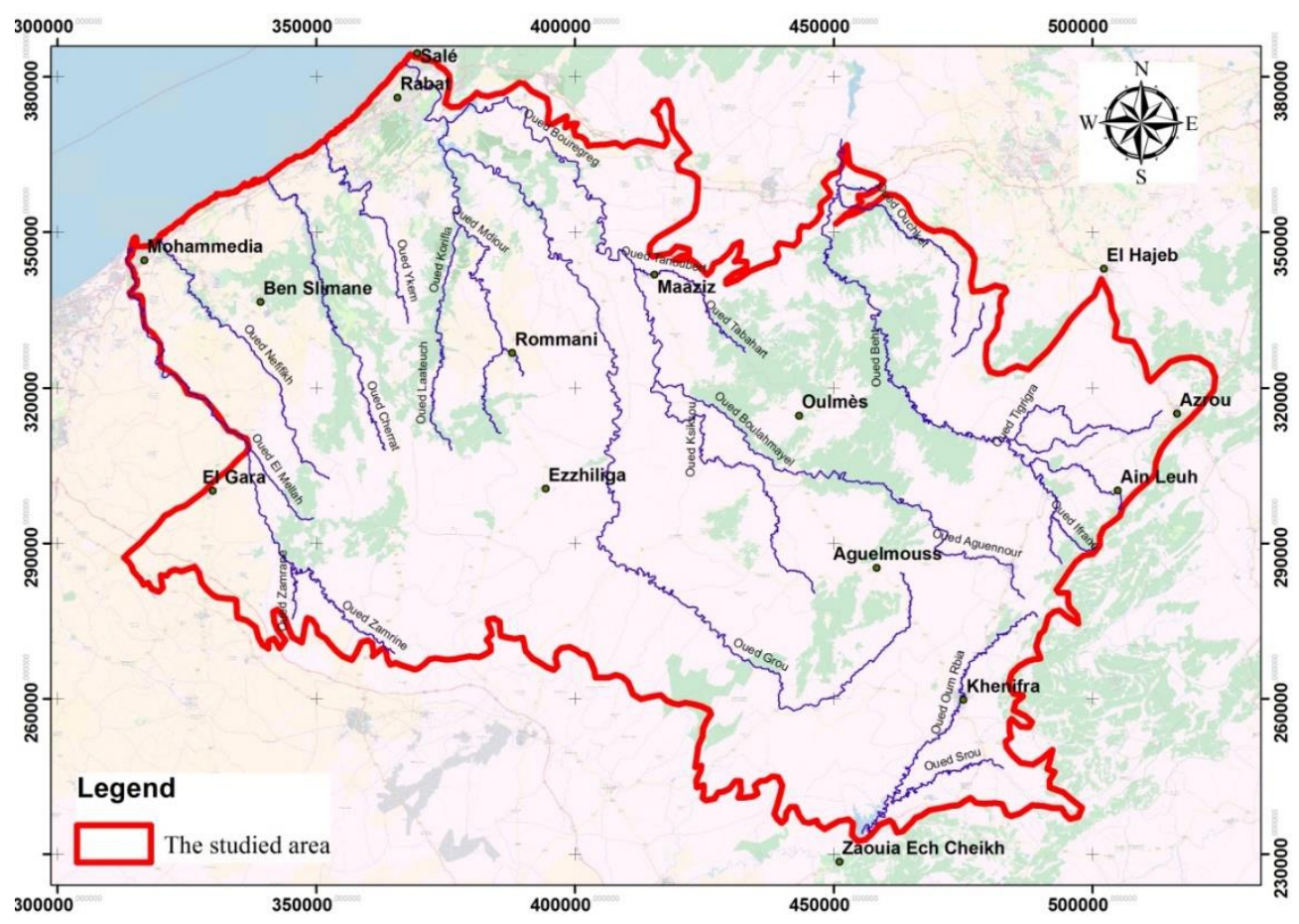

Figure 1. Location Map of the Studied Area

\section{Methodology}

The study was carried out from 2013 to 2016 . The methodology used is based on the implementation of more than 300 floristic surveys, covering the diverse Tetraclinis stands of the Central Plateau, data collection and analysis from all studies related to the region including the forest management plans and papers on the Moroccan Central Plateau flora (Fennane, 1988; Fennane, 2003; Chkhichekh et al., 2015; Dallahi et al., 2016a; Dallahi et al., 2016b).

-The study of the biologic types, according to Raunkiaer (1904), which are based on the place of the bud during seasons with adverse conditions are: Phanerophytes ( $\mathrm{Ph}$, Nanophanerophytes (Nph), Chamaephytes (Ch), Hemicryptophytes (He), Geophytes (G), and Therophytes (Th).

-Bioclimatic Zones: The different bioclimatic environments selected are those existing in the study area; meaning A: Aride; SA: Semi-aride; SH: Sub-humide, and $\mathrm{H}$ : Humide.

-Distribution within the Study Area: It describes the various biogeographic areas where the species in question is located. The studied biogeographic zones are: Moroccan Coastal Meseta (MCM) and Moroccan Central Plateau (MCP). 


\section{Result and Discussion}

The data compilation and analysis of the floristic surveys conducted in the field has allowed us to identify 233 taxa.

\section{Adoxaceae}

Viburnum tinus L.; Nph; SH, H; MCP.

\section{Amaranthaceae}

Atriplex halimus L.; Nph; A, SA; MCP.

Chenopodium murale L.; Th; SA, SH; MCM.

\section{Anacardiaceae}

Pistacia lentiscus L.; Nph; SA, SH, H; MCM and MCP.

Pistacia atlantica Desf.; Ph; A, SA, SH; MCM and MCP.

Rhus pentaphylla (Jacq.) Desf.; Nph; A, SA; MCM and MCP.

\section{Apiaceae}

Ammi majus L.; Th; A, SA, SH, H; MCP.

Bupleurum semicompositum L.; Th; A, SA, SH; MCP.

Daucus carota L subsp. Carota; Th; A, SA, SH, H; MCM and MCP.

Eryngium tenue Lam.; Th; SA, SH; MCP.

Eryngium tricuspidatum L.; He; A, SA, SH, H; MCM and MCP.

Eryngium triquetrum Vahl.; He; A, SA, SH; MCP.

Smyrnium olusatrum L.; He; SA-H; MCM and MCP.

\section{Apocynaceae}

Caralluma europaea (Guss.) N.E. Br.; Ch; A, SA, SH; MCP.

\section{$\underline{\text { Araceae }}$}

Arisarum vulgare Targ-Tozz.; G; A, SA, SH, H; MCM and MCP.

\section{Arecaceae}

Chamaerops humilis L.; Ch; A, SA, SH; MCM and MCP.

\section{Aristolochiaceae}

Aristolochia longa $\mathrm{L} . ; \mathrm{G} ; \mathrm{SA}, \mathrm{SH}, \mathrm{H}$; MCM.

Aristolochia baetica L.; Nph; SA, H; MCP.

\section{Asparagaceae}

Ornithogalum umbellatum L.; Ch; SA, SH; MCM.

Asparagus acutifolius L.; G; A, SA, SH; MCM and MCP.

Asparagus albus L.; Nph; A, SA, SH; MCM and MCP.

Asparagus altissimus Munby; Nph; S, A, SA; MCM and MCP.

\section{Asteraceae}

Atractylis cancellata L.; Th; A, SA, SH, H; MCP.

Calendula algeriensis Boiss. \& Reut.; Th; SA; MCM.

Carduus marianus L.; He; MCP.

Carlina corymbosa L.; He; SA, SH, H; MCM.

Carlina involucrata Poir.; Th; A, SA, SH, H; MCP.

Carlina racemosa L.; He; A, SA, SH; MCP.

Cichorium intybus L. subsp pumilum (Jacq.) Ball.; He; SA; MCM. 
Cirsium acarna (L.) Moench.; Th; MCP. Crepis vesicaria $\mathrm{L}$; $\mathrm{He}$; SA, SH; MCM.

Cynara humilis L.; G; A, SA, SH; MCM.

Cynara hystrix Ball.; G; MCP.

Echinops spinosus L.; He; A, SA, SH, H; MCP.

Evax pygmea (L.) Brot.; Th; A, SA, SH; MCM and MCP.

Filago gallica L.; Th; SA, SH, H; MCM and MCP.

Filago germanica $\mathrm{L}$.; Th; A, SA, SH, H; MCP.

Galactites tomentosa Moench; Th; SA, SH, H; MCM.

Hyoseris radiata L.; G; SA, SH; MCP.

Leontodon hispidulus (Del.) Boiss.; Th; A, SA, SH; MCM and MCP.

Leontodon saxatilis Lamk.; He; A, SA, SH; MCP.

Leontodon tuberosis L.; He; A, SA, SH; MCP.

Matricaria recutita (L.) Rauchert; Th; MCP.

Ormenis mixta (L.) Dumort.; Th; SA, SH; MCM and MCP.

Phagnalon saxatile (L.) Cass.; Ch; A, SA, SH, H; MCM and MCP.

Picris aculeata Vahl.; Ch; SA, SH; MCP.

Pulicaria odora (L.) Reichenb. var. typica Fiori.; He; SA, SH, H; MCM and MCP.

Scolymus hispanicus L.; He; A, SA, SH, H; MCM and MCP.

Senecio vulgaris L.; Th; SA, SH; MCP.

Silybum marianum L. Gaertn.; Th; SA, SH; MCP.

Sonchus asper (L.); Th; SA, SH, H; MCM and MCP.

Sonchus oleraceus L.; He; A, SA, SH, H; MCP.

Taraxacum officinale Weber.; He; MCP.

Tolpis barbata (L) Graetn.; Th; A, SA, SH, H; MCM and MCP.

Boraginaceae

Anchusa azurea Mill.; He; A, SA, SH, H; MCM.

Echium plantaginum L.; Th; A, SA, SH, H; MCM and MCP.

Brassicaceae

Biscutella didyma L. f. parviscutata Maire \& Weiller; Th; A, SA, SH; MCM and MCP.

Cardamine hirsuta L.; Th; SA, SH, H; MCM.

Diplotaxis catholica (L.) DC.; Th; A, SA, SH, H; MCM and MCP.

Trachystoma aphanoneurum (Maire \& Weiller); Th; SA; MCP.

\section{Campanulaceae}

Campanula dichotoma $\mathrm{L}$; $\mathrm{Th}$; SA, SH; MCP.

Campanula lusitanica L.; Th; SA, SH, H; MCP.

\section{Caprifoliaceae}

Knautia arvensis (L.) Coult.; He; MCP.

Lonicera arborea Boiss.; Ph; SH, H; MCP.

Lonicera implexa Aiton.; Nph; SA, SH, H; MCP. 


\section{Caryophyllaceae}

Cerastium dichotomum $\mathrm{L} . ; \mathrm{Th} ; \mathrm{MCP}$.

Cerastium glaucum Gren.; Th; SA, SH, H; MCP.

Cerastium glomeratum Thuill.; Th; SA, SH; MCP.

Herniaria glabra L.; He; SA, SH, H; MCM.

Paronychia argentea Lamk.; He; A, SA, SH, H; MCM and MCP.

Polycarpon tetraphyllum L.; He; A, SA, SH, H; MCP.

Silene gallica $\mathrm{L}$.; Th; SA, SH; MCM.

Spergula arvensis L.; Th; MCP.

\section{Cistaceae}

Cistus albidus L.; Nph; SA, SH, H; MCM and MCP.

Cistus ladaniferus L.; $\mathrm{Ch}$; $\mathrm{SH}, \mathrm{H}$; MCM and MCP.

Cistus monspeliensis L.; Nph; SA, SH; MCM.

Cistus salviifolius L.; Ch; SA, SH, H; MCM and MCP.

Cistus villosus L.; Ch; SA, SH, H; MCM and MCP.

Helianthemum guttatum (L.) Mill.; Th; SA, SH, H; MCM and MCP.

Helianthemum ledifolium (L.) Miller; Th; A, SA, SH, H; MCM.

\section{Convolvulaceae}

Convolvulus althaeoides L.; G; A, SA, SH, H; MCM.

Convolvulus siculus L.; Th; SA; MCP.

\section{Crassulaceae}

Sedum hirsutum All.; Ch; MCP.

Sedum sediforme (Jacq.) Pau.; He; MCP.

Umbilicus horizontalis (Guss.) DC.; G; A, SA, SH; MCP.

Umbilicus rupestris (Salisbury) Dandy.; He; MCP.

\section{Cucurbitaceae}

Bryonia dioica Jacq.; G; A, SA, SH, H; MCP.

\section{Cupressaceae}

Tetraclinis articulata (Vahl.) Masters.; Ph; SA, SH; MCM and MCP.

\section{Dioscoreaceae}

Tamus communis L.; G; SA, SH, H; MCM and MCP.

\section{Dipsacaceae}

Dipsacus sativus (L.) Honck.; He; MCP.

Ephedraceae

Ephedra fragilis Desf.; Nph; SA, SH; MCM and MCP.

\section{Ericaceae}

Arbutus unedo L.; Nph; SA, SH, H; MCP.

\section{Euphorbiaceae}

Euphorbia peplus L.; Th; A, SA, SH, H; MCM.

Mercurialis annua L.; Th; A, SA, SH, H; MCM and MCP.

Fabaceae

Anthyllis tetraphylla L.; Th; SA, SH, H; MCP. 
Astragalus lusitanicus L.; G; SA, SH; MCM and MCP.

Calycotome villosa (Poiret) Link; Ch; SH, H; MCM.

Ceratonia siliqua L.; Ph; SA, SH; MCP.

Coronilla scorpioides (L.) Koch.; Th; SA, SH, H; MCP.

Coronilla viminalis Salisb; Th; A, SA, SH; MCP.

Cytisus arboreus (Desf.) DC.; Nph; SA, SH, H; MCP.

Cytisus triflorus L'Hérit.; Ch; SH, H; MCP.

Genista linifolia L.; Nph; SA, SH; MCM.

Lotus arenarius Brot.; Th; MCP.

Lotus corniculatus L.; He; MCP.

Lotus creticus L.; He; MCP.

Medicago hispida Gaertn.; Th; SA, SH; MCM and MCP.

Medicago murex Willd.; Th; SA; MCP.

Medicago polymorpha L.; Th; A, SA, SH, H; MCM.

Medicago truncatula Gaertn.; Th; SA; MCM.

Ononis natrix L.; He; MCP.

Ononis pendula Desf.; Th; SA; MCP.

Ornithopus compressus L.; Ch; A, SA, SH; MCM and MCP.

Sarothamnus arboreus (Desf.) DC.; Nph; SA, SH, H; MCP.

Scorpiurus muricatus L.; Th; A, SA, SH; MCP.

Trifolium angustifolium L.; Th; A, SA, SH, H; MCM and MCP.

Trifolium arvense L.; Th; SA, SH, H; MCP.

Trifolium campestre Schreb.; Th; MCM.

Trifolium ochroleucon Huds.; He; MCP.

Trifolium stellatum L.; Th; SA, SH, H; MCM and MCP.

Vicia sativa L.; Th; SA, SH, H; MCP.

Vicia tenuifolia Roth.; Th; MCP.

Vicia tetrasperma (L.) Schreber.; Th; A, SA, SH, H; MCM and MCP.

\section{Fagaceae}

Quercus faginea Lamk.; Ph; SH, H; MCP.

Quercus rotundifolia $\mathrm{Lam}$.; $\mathrm{Ph}$; $\mathrm{SA}, \mathrm{SH}, \mathrm{H}$; MCM and MCP.

Quercus suber L; Ph; SA, SH, H; MCM and MCP.

\section{Gentianaceae}

Centaurium erythraea Rafn.; Th; A, SA, SH; MCP.

Centaurium maritimum (L.) Fritsch.; Th; SA, SH, H; MCP.

\section{Geraniaceae}

Erodium bipinnatum (Cav.) Willd.; Th; SA, SH; MCM and MCP.

Erodium chium (L) Willd.; Th; A, SA; MCM.

Geranium molle L.; Th; A, SA, SH, H.; MCM and MCP.

Geranium purpureum Vill.; Th; MCM.

\section{Lamiaceae}

Lavandula multifida L.; Ch; A, SA, SH; MCM and MCP. 
Lavandula stoechas $\mathrm{L}$; $\mathrm{Ch}$; $\mathrm{SA}, \mathrm{SH}, \mathrm{H} ; \mathrm{MCM}$ and MCP.

Ajuga iva (L) Schreb; He; A, SA, SH, H; MCP.

Ballota hirsuta Bentham; Nph; A, SA, SH, H; MCP.

Cleonia lusitanica (L.) L.; Th; A, SA, H; MCP.

Lamium flexuosum Ten.; He; H; MCP.

Prasium majus L.; Nph; A, SA, SH, H; MCM and MCP.

Teucrium decipiens Cosson \& Balansa.; Th; SA; MCP.

Teucrium fruticans L.; Nph; SA, SH, H; MCM and MCP.

\section{Liliaceae}

Allium pallens L.; G; SA, SH, H; MCP.

Linum strictum L.; Th; SA, SH, H; MCM and MCP.

Smilax aspera L.; Ph; SA, SH, H; MCM and MCP.

Urginea maritima (L.)Baker; G; A, SA, SH, H; MCM and MCP.

Malvaceae

Malva sylvestris L.; He; A, SA, SH, H; MCP.

Lavatera trimestris $\mathrm{L} . ; \mathrm{Th}$; MCM and MCP.

\section{Myrtaceae}

Myrtus communis L.; Nph; SA, SH, H; MCP.

\section{Oleaceae}

Jasminum fruticans L.; Nph; A, SA, H; MCM and MCP.

Olea europaea L. var oleaster; $\mathrm{Ph} ; \mathrm{A}, \mathrm{SA}, \mathrm{SH}, \mathrm{H} ; \mathrm{MCM}$ and MCP.

Phillyrea angustifolia $\mathrm{L}$; $\mathrm{Ph} ; \mathrm{SA}, \mathrm{SH}, \mathrm{H} ; \mathrm{MCP}$.

Phillyrea latifolia $\mathrm{L}$; $\mathrm{Ph} ; \mathrm{SA}, \mathrm{SH}, \mathrm{H} ; \mathrm{MCM}$ and MCP.

Phillyrea media $\mathrm{L} . ; \mathrm{Ph}$; SA, SH, H; MCM and MCP.

\section{Osmundaceae}

Osmunda regalis L.; He; MCP.

Papaveraceae

Fumaria macrosepala Boiss.; Th; MCP.

Papaver rhoeas L.; Th; A, SA, SH, H; MCP.

\section{Plantaginaceae}

Anarrhinum pedatum Desf.; He; A, SA, SH, H; MCM and MCP.

Globularia alypum L.; Ch; SA, SH; MCP.

Plantago coronopus L.; Th; A, SA, SH; MCM.

Plantago lagopus L.; Th; SA; MCM and MCP.

Plantago lanceolata L.; He; MCP.

Plantago mauritanicum Boiss. \& Reut.; Th; SA, SH, H; MCM.

Plantago ovata Forsk.; Th; A, SA, SH; MCP.

Plantago psyllium L.; Th; SA; MCM and MCP.

\section{Plumbaginaceae}

Limonium lobatum (L. fil.) Chaz.; Th; A, SA; MCM.

Limonium sinuatum (L.) Miller; Th; A, SA, SH; MCM and MCP. 


\section{Poaceae}

Aegilops ovata subsp triaristata (Wild) Roy; Th; SH; MCP.

Anthoxanthum odoratum L.; Th; SA, SH, H; MCP.

Aristida caerulescens Desf.; He; A, SA; MCP.

Arrhenatherum elatius (L.) Presl.; He; SA, SH, H; MCM.

Arundo donax L.; He; A, SA, SH, H; MCP.

Avena sterilis L.; Th; A, SA, SH; MCM and MCP.

Bellis sylvestris $\mathrm{Cyr}$;; Th; A, SA, SH, H; MCM.

Brachypodium phoenicoïdes (L.) Roem . \& Schultes.; Th; SA, SH; MCM.

Briza maxima L.; Th; SA, SH, H; MCM and MCP.

Briza minor L.; Th; SA, SH, H; MCP.

Bromus hordeaceus L.; Th; A, SA, SH, H; MCP.

Bromus mollis L.; Th; A, SA, SH, H; MCM and MCP.

Bromus rigidus Roth.; Th; A, SA, SH, H; MCM.

Bromus rubens L.; Th; A, SA, SH, H; MCM and MCP.

Cynodon dactylon (L.) Pers.; G; A, SA, SH, H; MCP.

Cynosurus elegans Desf.; Th; MCP.

Dactylis glomerata L.; He; A, SA, SH, H; MCM and MCP.

Festuca caerulescens Desf; He; A, SA, SH, H; MCM and MCP.

Hordeum murinum L.; Th; SA, SH; MCM.

Hyparrhenia hirta (L.) Stapf; He; SA, SH; MCM and MCP.

Koeleria pubescens (Lamk.) P. Beauv.; Th; SA, SH, H; MCM.

Lamarckia aurea (L) Moenc; Th; A, SA, SH; MCM and MCP.

Lolium multiflorum Lam.; Th; SA, SH; MCP.

Lolium rigidum Gaud; Th; A, SA, SH; MCM and MCP.

Melica ciliata L.; He; MCM and MCP.

Melica minuta L.; Th; MCP.

Oryzopsis miliacea (L.) Asch. \& Schweinf.; He; SA, SH, H; MCM.

Poa annua L.; Th; SA, SH, H; MCM.

Poa bulbosa L.; Th; SA, SH, H; MCP.

Stipa capensis Thumb.; Th; A, SA; MCP.

Stipa lagascae Roem. \& Schult.; Th; A, SA; MCM.

Trachynia distachya (L.) Link; Th; MCP.

Vulpia alopecuros (Schousb.) (Link.); Th; A, SA, SH; MCP.

Vulpia geniculata (L) Link Hort.; Th; A, SA, SH; MCM.

Vulpia myuros (L.) Gmel.; Th; A, SA, SH; MCM.

\section{Polygonaceae}

Rumex bucephalophorus L.; Th; A, SA, SH, H; MCP.

Polypodiaceae

Notholaena vellea (Aiton) Desv.; He; MCP.

Primulaceae

Anagallis arvensis L.; Th; A, SA, SH, H; MCP. 
Asterolinum linum-stellatum (L.) Duby.; Th; A, SA, SH, H; MCM.

\section{$\underline{\text { Ranunculaceae }}$}

Anemone palmata L.; G; SA, SH, H; MCP.

Clematis cirrhosa L.; Ph; SA, SH, H; MCM and MCP.

Ranunculus bulbosus L.; He; A, SA, SH, H; MCM.

Ranunculus paludosus Poiret.; He; SA, SH, H; MCP.

Resedaceae

Reseda alba L.; MCM and MCP.

Rhamnaceae

Rhamnus lyciö̈des L. subsp. oleoïdes (L.) Jah. Et Maire; Nph; SA, SH;

MCM and MCP.

Rhamnus lycioides L. subsp. atlantica; Nph; SA, SH; MCM and MCP.

Ziziphus lotus (L) Lam.; Nph; S, A, SA, SH; MCP.

\section{Rosaceae}

Crataegus monogyna Jacq.; Nph; SA, SH, H; MCP.

Rosa canina L.; Nph; MCP.

Sanguisorba minor Scop.; He; SA, SH; MCM.

\section{Rubiaceae}

Crucianella angustifolia L.; Th; SA, SH, H; MCP.

Galium mollugo L.; He; MCP.

Galium parisiense L.; Th; SA, SH, H; MCP.

Rubia peregrina L.; Ch; A, SA, SH, H; MCP.

Sherardia arvensis L.; Th; MCM.

\section{Rutaceae}

Ruta chalepensis L.; Ch; MCM and MCP.

\section{Santalaceae}

Osyris lanceolata Hochst. \& Steude; Nph; SA, SH, H; MCP.

\section{Selaginellaceae}

Selaginella denticulata (L.) Spring; He; MCM and MCP.

\section{Solanaceae}

Nicotiana glauca Graham; Nph; MCM

Withania frutescens (L.) Pauquy; Nph; SA; MCM and MCP.

\section{Tamaricaceae}

Tamarix africana Poir.; Nph; MCP.

Tamarix gallica L.; Nph; MCP.

\section{Urticaceae}

Parietaria mauritanica DR.; Th; A, SA, SH; MCP.

Urtica dioica L.; He; SA, SH; MCP.

Valerianaceae

Fedia pallescens Maire; Th; SH; MCP.

Verbenaceae

Vitex agnus-castus L.; Nph; A, SA, SH, H; MCP. 


\section{Vitaceae}

Vitis vinefera L.; Nph; SA, SH, H; MCP.

\section{Xanthorrhoeaceae}

Asphodelus microcarpus Salzm. \& Viv.; G; SA, SH; MCM and MCP.

The flora of Tetraclinis ecosystem in the Moroccan Central Plateau contains about 56 families. However, six important families contain almost $51 \%$ of the flora. However, these families are in order of importance: Poaceae (15\%), Asteraceae (14\%), Fabaceae (12\%), Lamiaceae (4\%), Caryophyllaceae (4\%), and Plantaginaceae (4\%).

Biological type analysis shows a dominance of Therophytes. This predominance is attributed to various factors such as the climate aridity of the region, associated with anthropogenic activities like grazing and deforestation. Indeed, the biological spectrum is typical of the semi-arid climate with a distribution percentage of $46 \%$ for Therophytes, $21 \%$ for Hemicryptophytes, $13 \%$ for Nanophanerophytes, $7 \%$ for Chamaephytes, $7 \%$ for Geophytes, and 6\% for Phanerophytes. associations:

Phytosociologically, these taxa are essentially integrated into the - $\quad$ Lonicero implexae-Tetraclinetum articulatae (Fennane, 1982),

- Phillyreo latifoliae-Oleetum sylvestris (Barbéro, Quézel \& RivasMartínez, 1981),

- Coronillo viminalis-Tetraclinetum articulatae (Barbéro, Quézel \& Rivas-Martínez, 1981),

- $\quad$ Phillyro mediae- Tetraclinetum articulatae (Fennane, 1982).

The phytosociological classification shows that these associations belong to the class of Quercetea ilicis Br.-Bl. 1947, the order of PistacioRhamnetalia alaterni (Rivas-Martínez, 1975) and the alliances of Tetraclini articulatae-Pistacion atlanticae (Rivas-Martínez et al., 1984) and AsparagoRhamnion oleoidis (Rivas-Martínez, 1975).

\section{Conclusion}

The floristic analysis of the Tetraclinis ecosystem in the Moroccan Central Plateau shows that species richness represents 5\% of the Moroccan vascular flora. The Moroccan Central Plateau is a home to an exceptionally rich floristic biodiversity, which is the result of its geographical position, orographic and edaphic characteristics, geological history, and its past and current climatic conditions. The protection of these taxa is needed more than ever, particularly those associated with Tetraclinis stands of the Central Plateau. However, it must be able to afford rigorous protection. Indeed, overgrazing and human pressure on the forest resources are likely to affect prejudicially this natural heritage. 


\section{References:}

1. Beaudet, G. (1969). Le plateau central marocain et ses bordures : étude géomorphologique. Thèse es-Lettres, Rabat, 478 p.

2. Benabid, A. (2000). Flore et écosystèmes du Maroc: Evaluation et préservation de la biodiversité. Ibis Press, Paris, 357 p.

3. Chkhichekh, A., El Aboudi, A., Aafi, A., Wahid, N. \& Benabid, A. (2015). A contribution to the knowledge of the Moroccan forest ecosystems: association of Quercetum rotundifolio-suberis ass. nova in the Central Plateau of Morocco. Plant Sociology 52 (2): 57-68.

4. Combe, Ferre, M. \& Thavin, J. (1975). Méseta centrale et côtière. Ressources en eau du Maroc, tome 2. ser. Géo. Maroc, Rabat.

5. Dallahi Y., El Aboudi A., Aafi A., (2016a). A Contribution to the Knowledge of Vascular Flora of the Site of Biological and Ecological Interest of Kharouba in the Central Plateau of Morocco. European Scientific Journal 12 (27): 358-365.

6. Dallahi, Y., El Aboudi, A. \& Aafi, A. (2016b). A contribution to the knowledge of the Moroccan Central Plateau plant communities. Plant Sociology 53 (2): 41-46.

7. Fennane, M. (2003). Inventaire des communautés végétales à l'aide du phytosociologue au Maroc. Ecologia Mediterranea 29: 87-106.

8. Fennane, M. (1988). Tetraclinisformations of Morocco: Syntaxonomy and key to associations. Documents Phytosociologiques 11: 303-310.

9. Raunkiaer, C. (1904). Types biologiques pour la géographie botanique. D.K.D. Vid Selsk. Over 2 : 347-437. 